\title{
COVID-19-associated liver injury (COVALI): role of hepatologists
}

\author{
Yoshio Sumida $^{1} \cdot$ Masashi Yoneda $^{1}$
}

Received: 8 April 2021 / Accepted: 28 June 2021 / Published online: 2 July 2021

(C) Japanese Society of Gastroenterology 2021

Keywords COVID-19 $\cdot$ Liver injury $\cdot$ Cirrhosis $\cdot$ SARSCoV-2

We read with great interest the article by $\mathrm{Li}$ and colleagues [1]. COVID-19 caused by severe acute respiratory syndrome coronavirus 2 (SARS-CoV-2), which has become a global pandemic. Elevated hepatic enzymes are common in patients with COVID-19, occurring in approximately $15-65 \%$ of SARS- CoV-2-infected individuals, particularly in severe cases. Liver biopsy results in patients with SARSCoV-2 have been characterized by non- specific findings, including steatosis, mild lobular and/or portal inflammation, and vascular pathology. It still remains unknown SARS-CoV-2 can directly infect hepatocytes and/or cholangiocytes. Mechanisms underlying COVID-19-associated liver injury (COVALI) are multifactorial and related to direct cytopathic effect of SARS-CoV-2, cytokine storm, hypoxia, abnormal coagulation, an administrated drug, and preexisting chronic liver diseases (CLD). The article extensively reviewed plausible mechanisms of COVALI. Overall, $2-11 \%$ of patients with COVID-19 were reported to have underlying CLD. Patients with cirrhosis have high rates of hepatic decompensation, acute-on-chronic liver failure and death from respiratory failure following SARS-

This comment refers to the article available online at https://doi.org/ 10.1007/s00535-021-01760-9.

Yoshio Sumida

sumida@koto.kpu-m.ac.jp

1 Division of Hepatology and Pancreatology, Department of Internal Medicine, Aichi Medical University, Nagakute, Aichi 480-1195, Japan
CoV-2 infection and should be prioritized for COVID-19 vaccination. The Asia Pacific Association for the Study of the Liver (APASL) launched a pan-Asia collaborative study "APASL COVID Liver Injury Spectrum (APCOLIS). APCOLIS study showed that the outcome was poor with Child-Pugh score 9 or more among 43 cirrhosis those exposed to SARS CoV2 infection [2]. According to data on 745 patients with CLD and SARS-CoV-2 from a UK hospital network. Baseline child stage and alcohol-related liver disease are independent risk factors for death from COVID-19 [3]. Accumulating evidences have proposed that metabolism-associated fatty liver disease may be a risk factor for COVID-19 severity. Liver transplant recipients do not appear to have an increased risk of mortality following SARS-CoV-2 infection compared with the matched general population. The effects of COVID-19 on underlying CLD require detailed evaluation, with further research warranted in this area. Remdesivir, an approved drug for patients with COVID-19, may have hepatotoxic effects. Remedesivir should not be used for COVI-19 patients with baseline liver enzymes more than five times the upper limits or decompensated cirrhosis. If patients with COVID19 receive dexamethasone or tocilizumab, HBV reactivation should be ruled out. International academic societies have recommended guidance outlining the evidence to date regarding the management of patients with COVID-19 and liver disorders, and CLD under the COVID-19 pandemic. The percentages of scheduled visits to screen HCC or varices in chronic hepatitis $\mathrm{C}$ after sustained virologic response declined rapidly after COVID-19 became pandemic in Japan [4]. We should avoid delay of HCC surveillance in CLD patients. If liver enzymes are commonly elevated in patients with COVID-19, we should first evaluate for the presence of other causes including acute viral infections (HAV, HBV, HEV, etc.) or pre-existing 
CLD, and strictly follow-up liver enzyme values. Hepatologists must play a certain important role in management of COVALI in collaboration with respiratory medicine and infectious disease specialists until efficacious SARS-CoV2 vaccines or effective medications are globally available [5].

\section{Declarations}

Conflict of interest The authors declare that they have no conflict of interest.

\section{References}

1. Li D, Ding X, Xie M, et al. COVID-19-associated liver injury: from bedside to bench. J Gastroenterol. 2021;56:218-30.
2. Sarin SK, Choudhury A, Lau GK, et al. Pre-existing liver disease is associated with poor outcome in patients with SARS CoV2 infection; the APCOLIS Study (APASL COVID-19 Liver Injury Spectrum Study). Hepatol Int. 2020;14:690-700.

3. Marjot T, Moon AM, Cook JA, et al. Outcomes following SARSCoV-2 infection in patients with chronic liver disease: an international registry study. J Hepatol. 2021;74:567-77.

4. Toyoda H, Yasuda S, Kiriyama S, et al. Impact of COVID-19 pandemic on surveillance of hepatocellular carcinoma: a study in patients with chronic hepatitis $\mathrm{C}$ after sustained virologic response. GastroHep. 2020;2:247-52.

5. Sumida Y, Kimoto S, Sakamoto K, et al. Relationship between COVID-19 and liver diseases: the role of hepatologists in clinical practice. Kanzo. 2020;61:8-15.

Publisher's Note Springer Nature remains neutral with regard to jurisdictional claims in published maps and institutional affiliations. 